\title{
Thermodynamic Performance of the 3-Stage ADR for the Astro-H Soft-X-ray Spectrometer Instrument
}

\author{
Peter J Shirron, Mark O Kimball, Bryan L James, Theodore Muench, Michael J DiPirro, Thomas \\ G Bialas, Gary A Sneiderman, Frederick S Porter and Richard L Kelley
}

NASA/Goddard Space Flight Center, Greenbelt, MD 20771

\begin{abstract}
The Soft X-ray Spectrometer (SXS) instrument[1] on Astro-H[2] will use a 3-stage ADR[3] to cool the microcalorimeter array to $50 \mathrm{mK}$. In the primary operating mode, two stages of the ADR cool the detectors using superfluid helium at $\leq 1.20 \mathrm{~K}$ as the heat sink[4]. In the secondary mode, which is activated when the liquid helium is depleted, the ADR uses a $4.5 \mathrm{~K}$ Joule-Thomson cooler as its heat sink. In this mode, all three stages operate together to continuously cool the (empty) helium tank and singleshot cool the detectors. The flight instrument - dewar, ADR, detectors and electronics - were integrated in 2014 and have since undergone extensive performance testing. This paper presents a thermodynamic analysis of the ADR's operation, including cooling capacity, heat rejection to the heat sinks, and various measures of efficiency.
\end{abstract}

\section{INTRODUCTION}

The ADR developed for $\left.\operatorname{SXS}^{3}\right]$ is unique in its ability to use two different heat sinks as a basis for cooling the $\mathrm{x}$-ray microcalorimeter array to $50 \mathrm{mK}$. The ADR is integrated within a cryogenic system $\left[{ }^{4}\right]$ that consists of a 30-liter superfluid helium tank (at 1.20 K), a 4.5 K Joule-Thomson (JT) cryocooler, and four 2-stage Stirling cryocoolers. A schematic of the cryogenic system is shown in Figure 1 and basic design parameters are given in Table 1. Two of the Stirling coolers pre-cool the JT cooler loop, and two are used to cool vapor cooled shields (an inner IVCS and an outer OVCS). The configuration of the ADR and cryogenic system creates redundancy in the ability to cool the detectors. The ADR and detectors can meet their performance requirements if either the liquid helium dewar or the JT cooler remain operational.

When operating with liquid helium, two of the ADR stages are used to single-shot cool the detectors while rejecting heat directly to the liquid. From a design perspective, this is a relatively standard application of a 2-stage ADR. Thermodynamic performance is easily modeled[5] using parameters such as the type and mass of the magnetocaloric material, the magnetic field used to drive the cycle, boundary temperatures, etc., and the mechanical design of the heat switches, suspension systems and detector assembly design parameters in order to determine heat loads.

When using the JT cooler in "cryogen-free" mode, the operation is constrained by the sensitivity of the detectors to changes in temperature and magnetic field in its vicinity. In particular, the detector array is supported inside a housing whose temperature must be kept very stable during science data collection. This housing is thermally connected to the helium tank, giving rise to a need for the ADR to actively stabilize the tank temperature. The only way to achieve the required temperature stability of both the detector array and its housing is to use two of the ADR stages to continuously cool the helium tank, and use the remaining stage, as before, to single-shot cool the detectors to $50 \mathrm{mK}$. Cooling the tank requires 
lifting not only the steady state heat loads incident on the tank, but heat generated by the ADR stages connected to it.

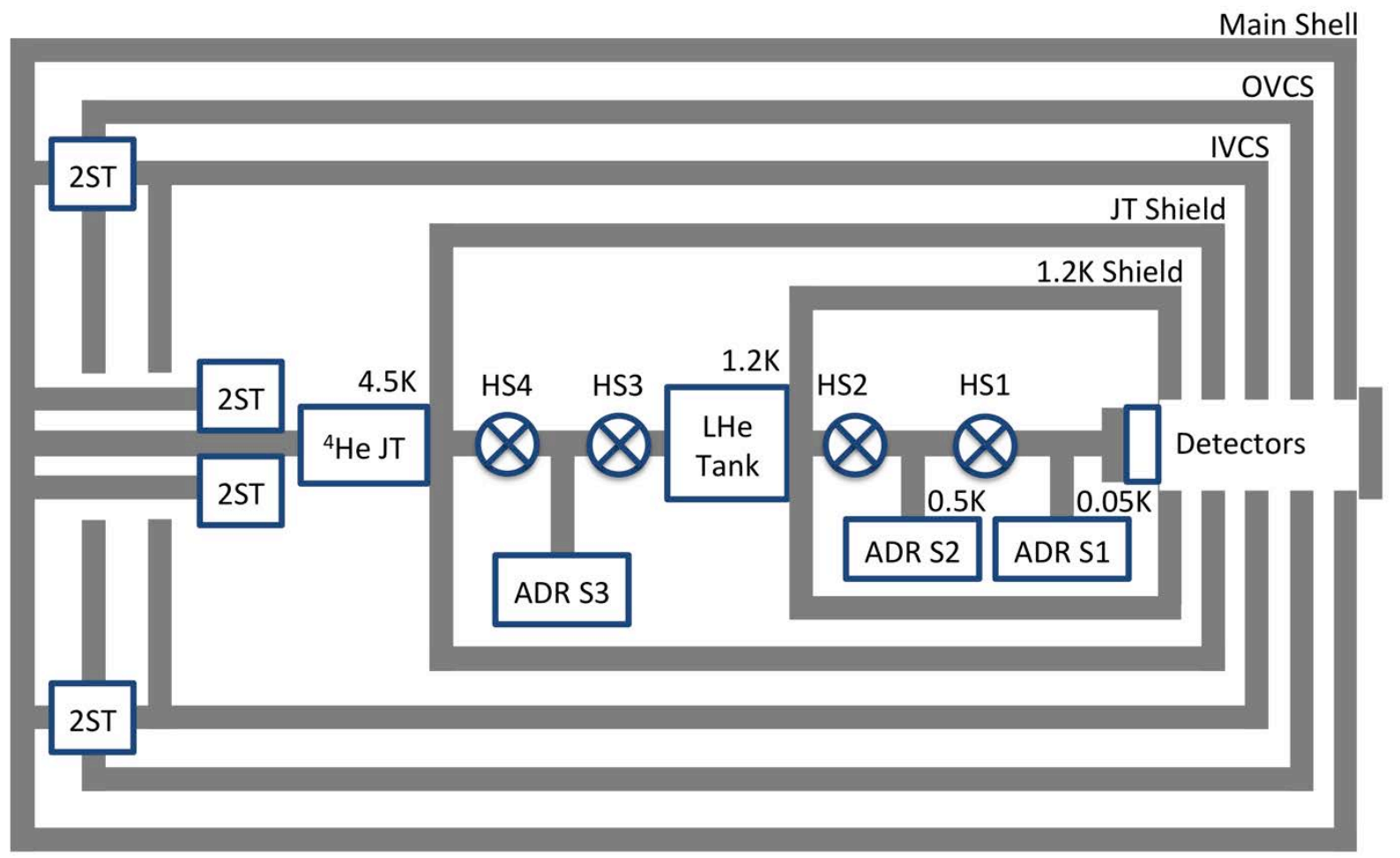

Figure 1. Schematic of the SXS cryogenic system.

Operation of the instrument in this mode requires the ADR to generate heat lift at least equal to the tank's parasitic load plus the load imposed by the $50 \mathrm{mK}$ stage, within the constraints of the JT cooler's ability to accept its rejected heat, and other constraints such as the physical space available and the maximum allowable ADR mass. The large number of interdependencies in the system could not be probed and optimized without a high-fidelity model of the ADR, helium tank and JT cooler that could simulate system performance. While many of the parameters on which such a model depends - such as thermal conductances of heat switches and thermal straps - can be estimated from mechanical designs, other quantities, such as the hysteresis heat generated by magnets and magnetic shielding, are not as readily determined.

In the design phase, the performance characteristics of each of the ADR's components were predicted using a variety of tools. In most cases, direct calculations (for example, salt pill cooling capacity) were combined with other experience-based assessments (such as the efficiency of heat absorption in a salt pill at low temperature) to arrive at performance estimates. The ADRs developed for the Astro-E and AstroE2 missions[6] provided a strong basis for many of these assessments. Scaling by appropriate parameters (mass, volume, magnetic field, etc.) allowed parameters such as magnet hysteresis heating and salt pill heat absorption efficiency at low temperature to be estimated. In some cases, adjustments were made where additional conservatism was deemed appropriate. As one example, the Astro-E and -E2 ADRs achieved approximately $75 \%$ heat absorption efficiency when operated at $60 \mathrm{mK}$. At the lower operating temperature of the SXS detectors $(50 \mathrm{mK})$, a more conservative efficiency of $70 \%$ was assumed. 
Despite the use of flight heritage components and systems as a design basis, a margin factor of $100 \%$ was applied to heat loads coming from the detectors, and parasitic loads from the suspension systems and heat switches. For heat exchange between stages, the only margin factor applied was the assumed heat absorption efficiency, since the heat of magnetization depends only on the mass and the magnetic field used in an ADR stage, and therefore has essentially no uncertainty associated with it.

Using the component characteristics and margin factors derived in this manner, a self-consistent ADR design was developed which met all of the SXS cooling requirements, in both operating modes. The system model was used not only to verify compliance with cooling requirements, but also to verify that the ADR met interface requirements in terms of peak and average heat rejection rates to the liquid helium and JT cryocooler. It also proved useful in developing control algorithms and operating parameters.

Performance testing of the engineering and flight model ADRs over the last several years have focused on evaluating a wide range of parameters, including thermal conductances, salt pill cooling capacities, magnet and shield hysteresis heating, and heat absorption efficiencies. Primarily this testing was conducted to verify the ADR meets its performance requirements, but a secondary goal was to characterize the system with enough resolution that even very small changes in performance, due possibly to aging, thermal cycling or environmental testing, could be identified. As a result, these measurements provide a basis for detailed evaluation of the thermodynamic performance of the ADR, which is presented in this paper. A by-product of this effort was a model of the ADR's heat output to the helium tank. With an accurate model, the regular cycling of the ADR can be used to track the mass of liquid helium and predict when it will run out on orbit. Results of that analysis are also presented.

Table 1. Design parameters of the SXS 3-stage ADR

\begin{tabular}{|l|c|c|c|}
\cline { 2 - 4 } \multicolumn{1}{c|}{} & Stage 1 & Stage 2 & Stage 3 \\
\hline Magnetocaloric material & $270 \mathrm{~g}$ CPA & $147 \mathrm{~g}$ GLF & 147 g GLF \\
Peak (average) magnetic field (T) & 2 & 3 & 3 \\
Maximum magnet current (amps) & 2 & 2 & 2 \\
Demagnetization temperature (K) & 0.8 & $\sim 1.5$ & 4.5 \\
Nominal operating temperature (K) & 0.05 & 0.5 & $\sim 1$ \\
\hline
\end{tabular}

\section{COOLING CAPACITY, HEAT REJECTION AND CYCLE EFFICIENCY IN CRYOGEN MODE}

Recycling of the ADR in cryogen mode is described in [7]. During the hold, Stage 1 stabilizes the detector array at $50 \mathrm{mK}$, while Stage 2 is stationed at $0.5 \mathrm{~K}$ to act as a heat intercept. As reported in $\left[^{7}\right]$, the heat load to $50 \mathrm{mK}$ stage is $0.86 \mu \mathrm{W}$ when the helium tank is $1.20 \mathrm{~K}$, and the hold time is 42.7 hours. (The total heat load scales approximately as $T_{\text {tank }}^{2.17}$.) The heat absorbed during the hold is $0.132 \mathrm{~J}$. Table 2 gives the current best estimate for the main contributors to the heat load, and the basis for the estimate, when the helium tank is $1.20 \mathrm{~K}$. The discrepancy between the estimates and total measured load is not fully understood.

Table 2. Current best estimates for heat loads to $50 \mathrm{mK}$ in cryogen mode (He tank at $1.20 \mathrm{~K}$ ). 


\begin{tabular}{l|llll}
\hline Component & Material & $A / L(\mathrm{~cm})$ & Heat load $(\mu \mathrm{W})$ & Reference $(\mathrm{s})$ \\
\hline Mechanical suspensions & Kevlar 49 & 0.0144 & 0.38 & 8,9 \\
Heat switch (HS1) & Ti15333 & 0.00514 & 0.017 & 10,11 \\
Detector array & Kevlar 49/CRES & & 0.25 & \\
\hline Total, estimated & & & 0.65 & \\
\hline Total, measured & & 0.86 & \\
\hline
\end{tabular}

As designed, Stage 1 is precooled to $0.8 \mathrm{~K}$ with the magnet fully charged, before being demagnetized to $50 \mathrm{mK}$. The magnet's design goal was an average field of $2 \mathrm{~T}$ at 2 amps, giving the 270 gram CPA salt pill a theoretical cooling capacity of $0.165 \mathrm{~J}$. Based on experience with the single-stage ADR built for Astro-E $\left[{ }^{6}\right]$, an efficiency factor of $70 \%$ was applied to yield a "usable" cooling capacity of $0.116 \mathrm{~J}$. The cooling capacity obtained in operation corresponds to $80 \%$ of the theoretical maximum.

Inefficiencies and reductions in cooling capacity can arise from a number of sources. The finite thermal conductance between the detector array and the salt cause the salt run colder than $50 \mathrm{mK}$, reducing its entropy capacity and decreasing the efficiency of heat absorption. The cooling of heat capacities within the salt pill, heat switch and detector system - especially stainless steel[12] - directly subtract from the salt's cooling capacity. In addition, these heat capacities can significantly lag the salt temperature during demagnetization and give up their heat across a large temperature gradient. Inhomogeneity in the magnetic field in the salt volume can lead to heat flow within the salt pill and excess entropy generation. And last, eddy current heating as the salt is cooled to $50 \mathrm{mK}$ and during the hold time can accumulate to a significant cooling loss. Salt pills are designed[13] to minimize these losses, but in a practical ADR they cannot be eliminated.

A final consideration is whether the magnet and shield combination produces the intended magnetic field. In the SXS ADR, Stage 1 was designed to produce $2 \mathrm{~T}$ at 2 amps. The design was based on magnetic field modeling in which the windings were represented by an average winding density fully filling the volume allocated to them. The as-wound magnets were terminated at a lower winding count to provide some clearance between the outer surface and the shield. This resulted in an average field to current ratio of $0.96 \mathrm{~T} / \mathrm{amp}$.

In an ideal ADR stage, the two entropy values that define its cooling capacity are the initial entropy at full field at the demagnetization temperature, $\mathrm{s}\left(B_{\max }, T_{\text {demag }}\right)$, and the final entropy at zero field at low temperature, $\mathrm{s}\left(0, T_{\text {low }}\right)$, where $T_{\text {low }}$ is the temperature of the detector array cooled by the ADR. The ideal cooling capacity is $\left(\mathrm{s}\left(0, T_{\text {low }}\right)-\mathrm{s}\left(B_{\text {max }}, T_{\text {demag }}\right)\right) \bullet T_{\text {low }}$.

In a real ADR, there are three entropies of interest, and how they differ from the ideal values provides information about which loss mechanisms are most important. They are the initial entropy, $\mathrm{s}\left(B_{\max }, T_{\text {demag }}\right)$, the entropy after demagnetization, $\mathrm{s}\left(B_{\text {max }}\right.$-hold,$\left.T_{\text {salt }}\right)$ and the final entropy at zero field, $\mathrm{s}\left(0, T_{\text {salt }}\right)$, where $T_{\text {salt }}$ is the temperature of the salt at low temperature, and $B_{\text {max }}$-hold is the magnetic field at the start of the hold. In the ideal case, the latter satisfies the relation $\mathrm{s}\left(B_{\text {max }}, T_{\text {demag }}\right)=\mathrm{s}\left(B_{\text {max-hold }}, T_{\text {low }}\right)$, giving $B_{\text {max-hold }}=0.118 \mathrm{~T}$.

The lower salt temperature reduces the cooling capacity to $\left(\mathrm{s}\left(0, T_{\text {salt }}\right)-\mathrm{s}\left(B_{\max }, T_{\text {demag }}\right)\right) \bullet T_{\text {salt }}$. While the salt's temperature is not measured directly, it can be inferred from fitting the current versus time during the hold time to standard demagnetization curves $\left[^{5}\right]$. The fit yielded a salt temperature of $48.5 \mathrm{mK}$, for which the maximum cooling capacity, using $B_{\max }=1.92 \mathrm{~T}$ and $T_{\text {demag }}=0.75 \mathrm{~K}$, is $0.160 \mathrm{~J}$. The measured capacity of 
$0.132 \mathrm{~J}$ corresponds to utilization rate, defined as the ratio of the measured cooling capacity to the ideal, of $82 \%$.

To better see where these losses are occurring, we compare the ADR's cooling capacity before and after demagnetizing to $50 \mathrm{mK}$. Demagnetization ends with $B_{\text {max } \text {-hold }}=0.113 \mathrm{~T}$ and $T_{\text {sal }}=0.0485 \mathrm{~K}$. The cooling capacity is then $\left(\mathrm{s}\left(0, T_{\text {salt }}\right) \mathrm{s}\left(B_{\text {max }- \text { hold }}, T_{\text {salt }}\right)\right) \bullet T_{\text {salt }}$, or $0.156 \mathrm{~J}$, giving a utilization rate of $84 \%$. The relatively small change, from $0.160 \mathrm{~J}$ to $0.156 \mathrm{~K}$, makes it clear that the salt pill is cooling efficiently during demagnetization (i.e. internal thermal gradients and eddy current heating are well controlled), and that the heat capacities of the components being cooled are small compared to that of the salt pill. A summary of cooling capacities and utilization rates is given in Table 3.

Table 3. Cooling capacities and utilization rates

\begin{tabular}{l|l|l|l|l|l|}
\hline \multirow{2}{*}{$A D R$} & \multicolumn{2}{|l|}{ Basis for computing cooling capacity } & $\begin{array}{l}\text { Cooling } \\
\text { Capacity }(J)\end{array}$ & Utilization & $\begin{array}{l}\text { Useable } \\
\text { Cooling } \\
\text { Capacity }(J)\end{array}$ \\
\cline { 2 - 5 } & Starting point & Ending point & & $100 \%$ & 0.165 \\
\hline Ideal & $2 \mathrm{~T}, 0.8 \mathrm{~K}$ & $0 \mathrm{~T}, 50 \mathrm{mK}$ & 0.165 & $70 \%$ & 0.115 \\
\hline As designed & $2 \mathrm{~T}, 0.8 \mathrm{~K}$ & $0 \mathrm{~T}, 50 \mathrm{mK}$ & 0.165 & $82 \%$ & 0.132 \\
\hline As built/operated & $1.92 \mathrm{~T}, 0.75 \mathrm{~K}$ & $0 \mathrm{~T}, 48.5 \mathrm{mK}$ & 0.160 & $84 \%$ & 0.132 \\
\hline As built/operated & $0.113 \mathrm{~T}, 48.5 \mathrm{mK}$ & $0 \mathrm{~T}, 48.5 \mathrm{mK}$ & 0.156 & & \\
\hline
\end{tabular}

\section{ADR Heat Sources}

An important aspect of a flight cooler is its overall efficiency. To assess the ADR's efficiency, it is first necessary to determine the heat output during a full refrigeration cycle. There are three main components to the heat rejection: heat of magnetization of the salt pill(s), power dissipated by heat switches, and hysteresis from magnets and magnetic shielding. Other contributions such as from eddy current heating may be relevant when considering the heat absorbed by the salt pills at low temperature, but are a negligible contribution to the heat flow to the sink.

\section{Salt Pill Heat of Magnetization}

For the analysis presented here, heat of magnetization from the salt pills is determined by integrating the heat flow through the heat switch(es) that connect the salt pills to the sink. The on-state conductance is determined by observing the temperatures across the switch in the presence of a known heat flow, which can be generated either by a heater on the cold end of HS1, or by magnetizing the Stage 1 salt pill. The heater can only be powered by ground support electronics that were connected only during preliminary testing after integration. During that time, both methods of determining conductance were used in order to verify that calculated heat flow from the salt pill could subsequently be used to calibrate the conductance. The agreement was within 1\%. For the gas-gap heat switches [14], the on-state conductance is proportional to temperature. Measuring it at one fixed point is enough to be able to track it during subsequent operations.

\section{Heat Switch Power}

Power used to actuate a heat switch can usually be calculated from power supply settings and actuator resistance. For example, gas-gap switches are typically powered using a resistive heater attached to the 
getter; one can readily determine the power applied. For SXS, the situation is more complicated, as the getter is temperature controlled using a feedback loop, and the instantaneous power is not directly accessible. Instead, previous measurements of the getter temperature versus applied power are used to convert instantaneous temperature readings to applied power.

The getters in the SXS switches were specially designed to require very low power for actuation - in the range of 0.3 to $0.5 \mathrm{~mW}$ - making the total heat dissipated by the heat switches a relatively small fraction of the total ADR output.

\section{Magnet and Magnetic Shield Hysteresis}

A significant source of the heat expelled during an ADR cycle comes from dissipation in the magnets and magnetic shields. In the magnets, flux motion into and out of the NbTi wire - a type II superconductor dissipates heat, and in the magnetic shields, hysteresis in the soft ferromagnetic material generates heat as the ADR undergoes large-scale magnetization and demagnetization.

For reference, the magnets are wet wound using multifilamentary $\mathrm{NbTi}$ wire with a $\mathrm{Cu}$ to superconductor ratio of $1.4: 1[15]$. The bare wire diameter is $0.076 \mathrm{~mm}$, and $0.101 \mathrm{~mm}$ with insulation. The packing density is $10^{4}$ per $\mathrm{cm}^{2}$. The shields are Vanadium permendur (also known as Carpenter Hiperco 50), annealed for maximum flux density. The outer profile is tailored so that the shield just approaches saturation in the mid-section at peak current. Additional details are included in Table 2.

Heat generation in the magnets and shields (shown in Figure 2) depends on local field densities and the rate of field change. Modeling this dissipation would require knowledge of material properties (permeability of the vanadium permendur, and magnetization of CPA and GLF as a function of magnetic field and temperature) to better precision than currently exists. Instead, for this analysis, we undertook direct calorimeters measurements of the heat generated by each of the ADR's three magnets and shields when ramped over their full range. For each stage, the heat switches were configured to prevent heat of magnetization in the salt pills from affecting the measurement.

For stages 1 and 2, each magnet was ramped separately with heat switches 1 and 2 powered off, when the dewar was full of superfluid helium. Both of these stages are mounted so that any heat from the magnets and shields is directly absorbed by the liquid helium. By monitoring the temperature of the liquid helium as each stage was ramped from zero to full $(2 \mathrm{amp})$ current and then back to zero, the instantaneous heating rate as a function of current could be determined, for both increasing and decreasing current. The temperature response of the liquid helium was calibrated by imposing a known $(0.1 \mathrm{~W})$ heat flow for 100 seconds. Due to the extremely high effective thermal conductivity of superfluid helium, the constant heat input lead to a perfectly linear temperature rise over time. The slope could then be used to translate temperature changes during magnet ramping to heating rates.

For Stage 3, similar ramps were performed, with heat switches 3 and 4 in the off state, while monitoring the heat flow across the thermal strap to the JT cryocooler. The conductance of that strap was calibrated by imposing a known heat flow from the ADR to the JT cooler.

The nature of hysteresis heating suggests, and in practice we have observed, that the amount of heat generated in a magnet and shield, Qhysteresis, as current is steadily ramped (either up or down), depends only on the change in current and is independent of the ramp rate. Confirmation of this rules out eddy currents as a significant source of heating. Consequently, the heat generation rate is proportional to the ramp rate $(\mathrm{dI} / \mathrm{dt})$ or, equivalently, to the applied voltage via $\mathrm{V}=\mathrm{L}(\mathrm{dI} / \mathrm{dt})$, and hence the ratio of the heating rate to applied voltage depends only on current. 

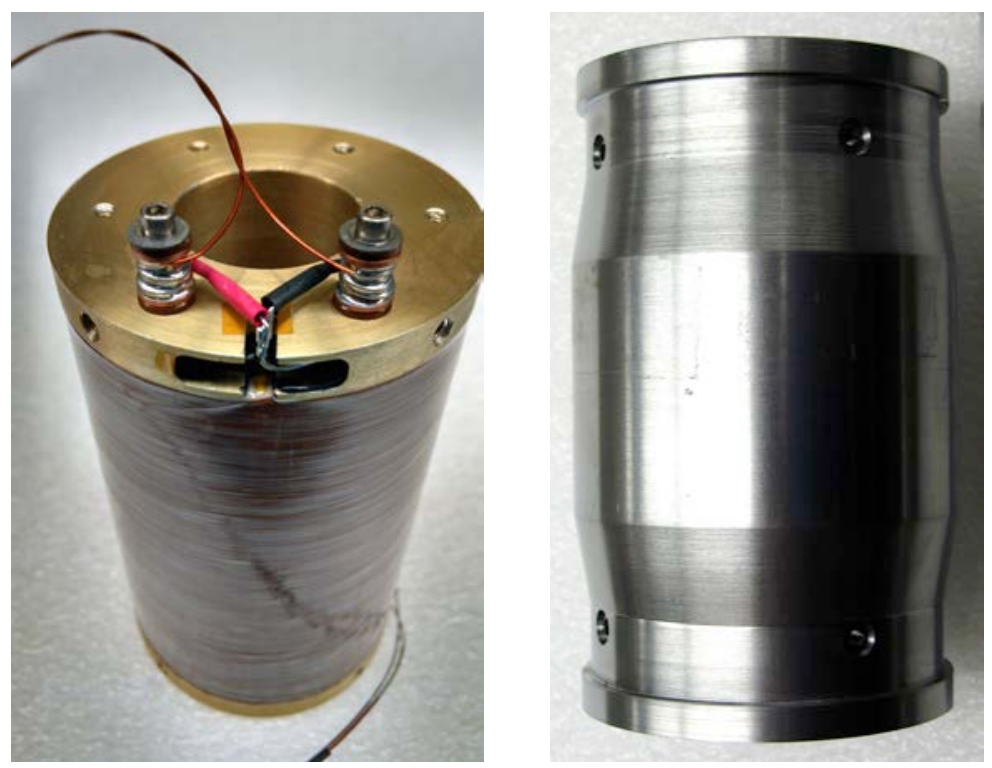

Figure 2. Magnet and vanadium permendur shield (end caps not installed) for Stages 2 or 3.

Figure 3 shows the heat generated in each of the three magnet/shield combinations per unit change in absolute value of current, for both increasing and decreasing current. Instantaneous heating rates can be obtained by multiplying $\mathrm{dQ}_{\text {hysteresis }} / \mathrm{dI}$ by the absolute value of the instantaneous magnet voltage and dividing by inductance, $\mathrm{L}$. By integrating $\mathrm{dQ}_{\text {hysteresis }} / \mathrm{dI}$ over current, the heat generated in a sweep can be calculated. Along with relevant magnet parameters, Table 4 presents the total heat generation for full ramps in current of 0 to $2 \mathrm{amps}$, and 2 to 0 amps. The total heat generated for complete cycles (0 to 2 amps to 0 ) for the three stages are $2.18 \mathrm{~J}, 2.15 \mathrm{~J}$ and $1.80 \mathrm{~J}$, respectively.

Table 4. Magnet and shield parameters.

\begin{tabular}{l|ccc}
\hline & Stage 1 & Stage 2 & Stage 3 \\
\hline Magnet winding volume $\left(\mathrm{cm}^{3}\right)$ & 190.8 & 139.8 & 139.8 \\
NbTi volume $\left(\mathrm{cm}^{3}\right)$ & 44.7 & 32.8 & 32.8 \\
Average magnetic field in bore (T) & 2 & 3 & 3 \\
Field to current ratio (T/A) & 1 & 1.5 & 1.5 \\
Inductance (H) & 256 & 205 & 204 \\
Magnetic shield volume $\left(\mathrm{cm}^{3}\right)$ & 423 & 253 & 239 \\
\hline Magnet operating temperature (K) & 1.2 & 1.2 & 4.5 \\
\hline Hysteresis heat during 0-2 amp ramp (J) & 0.94 & 0.99 & 0.75 \\
Hysteresis heat during 2-0 amp ramp (J) & 1.24 & 1.16 & 1.05 \\
Hysteresis heat for full cycle (0-2-0 amp) (J) & 2.18 & 2.15 & 1.75 \\
\hline
\end{tabular}



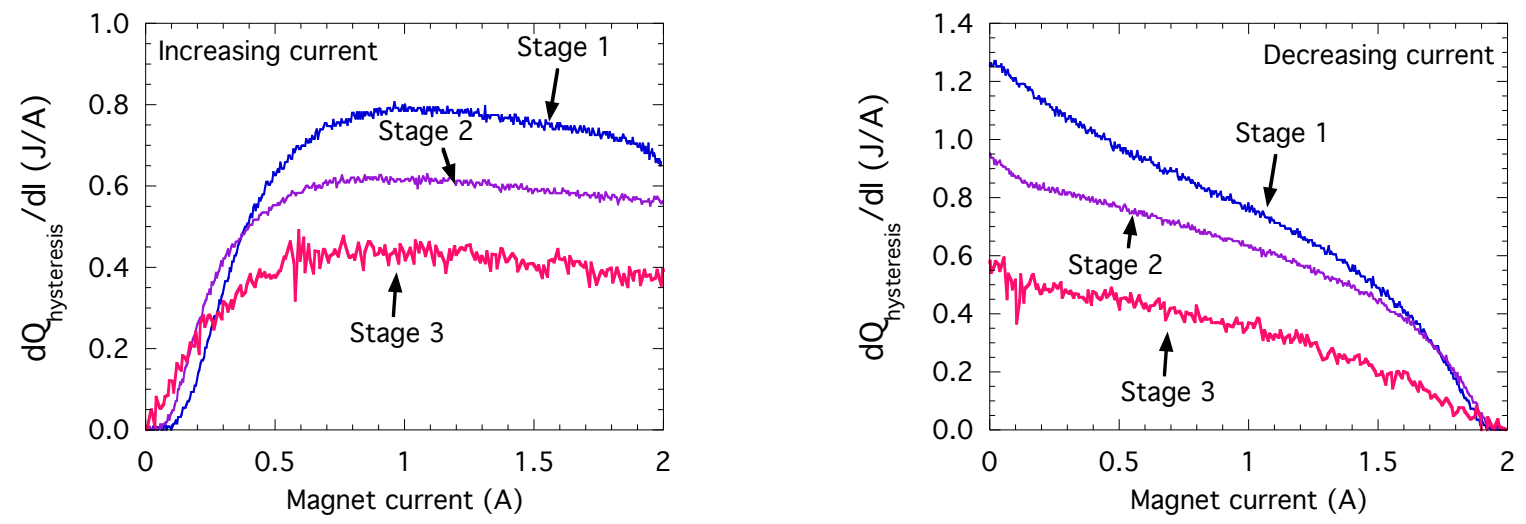

Figure 3. Hysteresis heat generation per change in absolute value of magnet current for Stages 1, 2 and 3 when the current is (left) increasing and (right) decreasing.

\section{ADR Heat Rejection During a Complete Cycle}

The three contributions (salt pill heat, getter dissipation, and hysteresis heat) described above can be reconstructed from real-time readings of various temperatures and the magnet charging voltages. Figure 4 shows the instantaneous heat output during a recycle that occurs at the end of a hold time. Integrating gives individual totals of $7.67 \mathrm{~J}, 0.79 \mathrm{~J}$ and $4.11 \mathrm{~J}$, respectively, and a sum of $12.57 \mathrm{~J}$. During the subsequent hold time, an additional $0.14 \mathrm{~J}$ is generated from hysteresis, giving a total heat output of 12.71 J every (approximately) 43.5 hours. This translates to a time average heat load on the liquid helium of $0.081 \mathrm{~mW}$.

If the ADR is cycled on a shorter time scale, for example, every 24 hours during ground passes, the salt pill and heat switch dissipation are proportionally smaller, but the amount of hysteresis heat generated is essentially unchanged since both magnets still undergo the excursion to full current and back to the same starting currents at the beginning of the hold time. In this scenario, each cycle will imposed slightly less than $10 \mathrm{~J}$ on the helium, for a time average heat load of $0.115 \mathrm{~mW}$.

The thermal budget for the helium dewar allows the time average load from the ADR to be as high as $0.25 \mathrm{~mW}$. Even if the ADR is recycled more often - resulting in proportionally less salt pill heat and getter dissipation, but the same hysteresis heat - the average load is still well within the budget. 


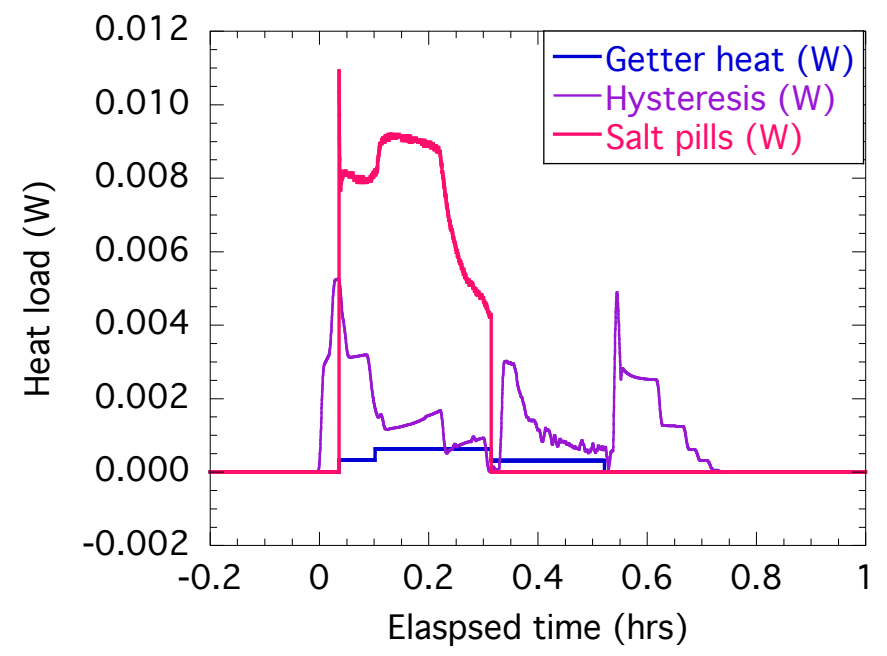

Figure 4. Instantaneous heat flows during an ADR recycle in cryogen mode. Time is zero at the beginning of the recycle.

\section{ADR Efficiency}

The most direct measure of the ADR's efficiency is the ratio of entropy absorbed by the cold stage as a fraction of the entropy expelled to the sink: $\varepsilon=\left(\dot{Q}_{\text {in }} / T_{\text {low }}\right) /\left(\dot{Q}_{\text {out }} / T_{\text {sink }}\right)$. For the results presented here, the efficiency is $25 \%$. This formulation does not differentiate between heat absorbed from parasitic sources (heat switches and Kevlar suspensions) and that coming from the detector array - i.e. "useful" cooling - although we note that it also does not count the entropy lifted by Stage 2 .

We justify this approach on the basis that while the total heat load to $50 \mathrm{mK}$ is known, the individual contributions are not. The ADR and detector array have only been tested in a flight-like configuration as a complete unit. Earlier measurements with the ADR only were not in similar enough conditions to allow the difference in heat loads to be ascribed to the detectors. It is therefore not possible to identify the portion of the cooling capacity that is used for detector cooling. Instead, this efficiency is useful for computing heat output from an ADR on the basis of its total cooling capacity.

\section{ADR Heat Output as a Probe of Helium Mass}

With the helium tank at superfluid conditions, it possible to use the heat output of the ADR as a probe of the liquid mass - and consequently to validate the heat output calculation. By measuring the temperature response of the tank to a known heat load (from a heater coil on the bottom of the tank), the heat output during a recycle can be converted to a rate of temperature change. Since the three contributions to the ADR's heat output appear at different times, a match between the shape of the two curves would confirm that the individual contributions and the aggregate are correct.

Figure 5 gives a comparison of the measured temperature of the helium tank to that obtained by integrating the rate of temperature change calculated from heat flow. The calculated temperatures include a cooling term arising from an increase in pumping rate due to the increase in tank pressure (a constant negative heating load is applied starting at the mid-point in the recycle). The shape of the curves is an excellent match, and in fact the discrepancies - for example at 0.6 hours where the calculated temperature rises faster than the actual - comport with the fact that the hysteresis heat generated at those times is conducted through the magnet and shields, and one would expect the actual temperature to lag. 


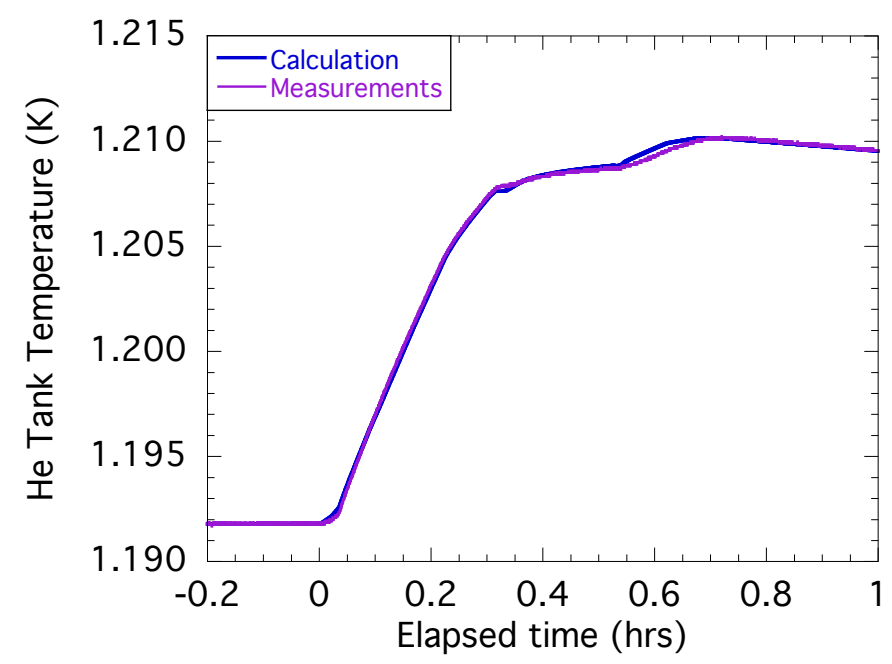

Figure 5. Comparison of the helium tank during an ADR recycle obtained from direct measurement and as calculated from ADR heat flow.

Many ADR recycles have been analyzed as a mass gauge probe, at a variety of tank fill levels. Comparing those associated with secondary heat pulse mass gauge measurements[16] consistently gave agreement to within \pm 1 liter. In cases where ADR cycles were performed multiple times at regular intervals - with negligible mass loss in between - the individual mass values were consistently within 1 liter of the average, independent of fill level. These results are taken as a confirmation that the model accurately reproduces the heat generation.

\section{CRYOGEN-FREE MODE OPERATION}

\section{Cooling Power and Cooling Capacity}

In cryogen-free mode $\left.{ }^{7}\right]$, the ADR produces continuous cooling of the tank at $\sim 1.5 \mathrm{~K}$, and detector cooling at $50 \mathrm{mK}$. Recycling of Stage 1 involves a different sequences of steps from cryogen mode, but the end condition is the same: the salt pill is cooled to $0.8 \mathrm{~K}$ at full field $(1.92 \mathrm{~T})$. Hence the theoretical cooling capacity is the same as given previously, $0.165 \mathrm{~J}$.

Table 5 lists the estimated and measured loads. The main difference in cryogen-free mode is that Stage 2 is stationed at $\sim 1.5 \mathrm{~K}$, rather than $0.5 \mathrm{~K}$, significantly increasing the heat leak through heat switch HS1. Figure 6 shows the expected heat load as a function of tank temperature, with values measured at two points.

Table 5. Current best estimates for heat loads to $50 \mathrm{mK}$ in cryogen-free mode (He tank at $1.525 \mathrm{~K}$ ).

\begin{tabular}{l|llll}
\hline Component & Material & $A / L(\mathrm{~cm})$ & Heat load $(\mu W)$ & Reference $(\mathrm{s})$ \\
\hline Mechanical suspensions & Kevlar 49 & 0.0144 & 0.62 & 8,9 \\
\hline
\end{tabular}




\begin{tabular}{|c|c|c|c|c|}
\hline Heat switch (HS1) & Ti15333 & 0.00514 & 0.83 & 10,11 \\
\hline Detector array & Kevlar/CRES & & 0.35 & \\
\hline Total, estimated & & & 1.80 & \\
\hline Total, measured & & & 2.29 & \\
\hline
\end{tabular}

The demonstrated hold time is 15.1 hours, giving a total heat absorption of $0.125 \mathrm{~J}$. The lower utilization rate $(80 \%)$ is consistent with the lower salt temperature of $47.5 \mathrm{mK}$, as determined from fits to demagnetization curves.

During Stage 1's hold time, Stages 2 and 3 work cooperatively to continuously cool the helium tank. Stage 3 cycles continually to transfer heat from the tank to the JT cooler. Stage 2 is thermally connected to the tank through heat switch HS2, enabling it to absorb heat form the tank while Stage 3 is warm, and to push that heat back out when Stage 3 is cold. A key detail is that it pushes out more heat each time than it absorbs, gradually building up the cooling capacity that is needed to recycle Stage 1.

Stage 3 provides a gross heat lift, $\dot{Q}_{i n}$, which can be calculated from cycle parameters (Eqn 1). $n_{\text {salt }}$ is the salt mass (in moles), $\tau_{c y c l e}$ is the cycle period, and $\varepsilon_{i n}$ is the efficiency of heat absorption (or utilization rate).

$$
\dot{Q}_{\text {in }}=\left[\left(S\left(B_{\text {max }}, T_{\text {demag }}\right)-S\left(0, T_{\text {low }}\right)\right) T_{\text {low }}\right] n_{\text {salt }} \varepsilon_{\text {in }} / \tau_{\text {cycle }}
$$

The term in square brackets is the cooling capacity at $T_{\text {low }}$, the temperature of the stage as it absorbs heat from the helium tank. Typically $T_{\text {low }}$ is regulated to be $0.9 \bullet T_{\text {tank }}, B_{\max }$ is $2.6 \mathrm{~T}$, and $T_{\text {demag }}$ is $4.7 \mathrm{~K}$. Using 147 grams of GLF, the cooling capacity when the tank is $1.625 \mathrm{~K}$ is $3.83 \mathrm{~J}$. The cycle period is consistently 23 minutes, at which the cooling power is $\varepsilon_{i n} \bullet(2.78 \mathrm{~mW})$.

To determine the efficiency, $\varepsilon_{i n}$, we compare the actual heat lift to this expected value. There are five contributions to the heat that Stage 3 must lift: 1) the parasitic heat load on the He tank, 2) hysteresis from Stage 2 magnet, plus a small contribution from Stage 1,3) power dissipated by HS2, 4) the inefficiency associated with moving heat into and out of Stage 2, and 5) charging of Stage 2. Another potentially significant heat source is eddy current heating in Stage 3's salt pill. The salt pill is specifically designed to minimize eddy currents, but they have not been independently quantified. For this analysis, their effect, if any, is included in the efficiency.

Hysteresis and heat switch dissipation are computed as before from magnet charging voltages and the temperature of HS2's getter. The parasitic heat load of $0.65 \mathrm{~mW}$ on the tank is known from measurements of the steady state boiloff rate[17] adjusted for an average JT shield temperature of $4.5 \mathrm{~K}$. The two remaining heat flows are obtained by integrating the heat flow across heat switch HS2. Initially, Stage 2 is allowed to give off excess heat so that it builds cooling capacity. When its field approaches the maximum, Stage 3's cooling power is throttled to stabilize the current (averaged over a cycle). In that latter mode, Stage 2's entropy remains at a constant average value, and the non-zero (integrated) heat flow across HS2 is the het load representing Stage 2's inefficiency. Subtracting this from the (integrated) heat flow as Stage 2 builds cooling capacity gives Stage 2's charging rate.

Table 6 summarizes the heat loads lifted by Stage 3 in cryogen-free operation at $1.625 \mathrm{~K}$. A similar analysis was performed when the helium tank was held at $1.525 \mathrm{~K}$. The only significant change is the charging rate of Stage 2, as it reflects the decreased capability of Stage 3 to lift heat. The two points are 
shown in Figure 6 compared to the cooling power calculated one obtains from Eqn 1 with $\varepsilon_{i n}=1$. The inferred utilization rate is $\varepsilon_{i n}=72-79 \%$ in the $1.525-1.625 \mathrm{~K}$ range.

Table 6. Estimated and measured heat loads in cryogen-free mode with He tank at $1.625 \mathrm{~K}$.

\begin{tabular}{l|c}
\hline Heat source & Time average value $(\mathrm{mW})$ \\
\hline He tank parasitic & 0.65 \\
Heat switch dissipation & 0.34 \\
Hysteresis & 0.22 \\
Stage 2 inefficiency & 0.23 \\
Stage 2 charging & 0.59 \\
\hline Total & 2.20 \\
\hline
\end{tabular}

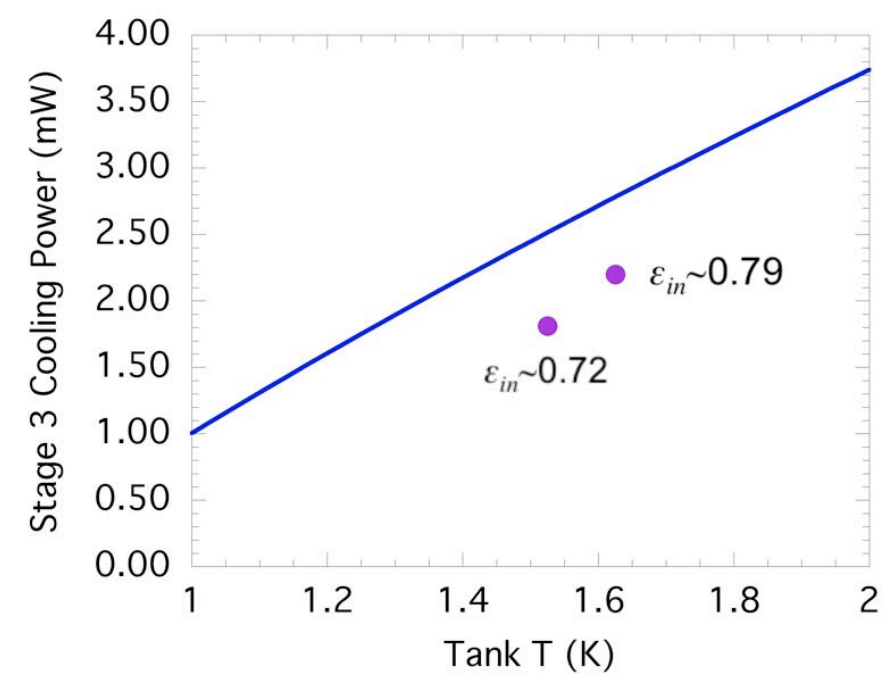

Figure 6. Computed Stage 3 cooling power at 1.525 and $1.625 \mathrm{~K}$ compared to the ideal curve.

The efficiency of Stage $3, \varepsilon_{S 3}$, can be computed as

$$
\frac{\dot{Q}_{\text {in }}}{\dot{Q}_{\text {out }}}=\varepsilon_{S 3}\left(\frac{T_{\text {tank }}}{T_{J T}}\right)=\left(\varepsilon_{\text {in }} \frac{T_{J T}}{T_{\text {high }}} \frac{T_{\text {low }}}{T_{\text {tank }}}\right) \frac{T_{\text {tank }}}{T_{J T}}
$$

where $T_{\text {tank }}$ and $T_{J T}$ are the temperatures of the He tank and JT cooler, and $T_{\text {low }}$ and $T_{\text {high }}$ are the setpoints for absorbing and rejecting heat. For the case of $T_{\text {tank }}=1.525 \mathrm{~K}$, the default value for on orbit operations, $T_{J T} \sim 4.5 \mathrm{~K}, T_{\text {low }} \sim 1.3 \mathrm{~K}$, and $T_{\text {high }} \sim 4.7 \mathrm{~K}$, the demonstrated efficiency for lifting $1.8 \mathrm{~mW}$ from $1.525 \mathrm{~K}$ to $4.5 \mathrm{~K}$ is $59 \%$. Of the $1.8 \mathrm{~mW}$, however, only $1.14 \mathrm{~mW}$ can be considered "useful" cooling since all other terms are internal losses - giving an aggregate efficiency of $37 \%$.

\section{SUMMARY}


The ADR built for the SXS instrument on Astro-H was designed to meet a challenging set of requirements, driven by the need to operate with full redundancy using either superfluid helium or a $4.5 \mathrm{~K}$ JT cryocooler as its heat sink. It is the first space-qualified ADR to include continuous cooling as part of its normal operating modes. The design was established using conservative assumptions for heat absorption efficiencies and internal parasitic heat generation, and a high-fidelity model was developed to predict performance and to aid in developing control algorithms.

Through a lengthy test campaign, the ADR's thermodynamic performance has been extensively characterized in both cryogen and cryogen-free modes. The analysis presented here focuses on comparing the "ideal" and "usable" cooling capacities of the stage that single-shot cools the detectors to $50 \mathrm{mK}$, in order to determine the utilization rates. The rates are, as expected, found to depend on the heat load and the gradients that exist between the heat sources and the salt. In cryogen mode, the heat load to $50 \mathrm{mK}$ is $0.86 \mu \mathrm{W}$, and the utilization rate is $84 \%$. In cryogen-free mode, the heat load is $2.2 \mu \mathrm{W}$ and the rate is $80 \%$. Both are significantly higher than the value of $70 \%$ assumed during the design phase.

Heat rejection from the ADR has been characterized by a variety of calorimetric measurements. By calibrating the helium tank's response to a known heat input, or using thermal straps as heat meters, it was possible to measure the hysteresis heat per full cycle of each magnet, and of the ADR in both operating modes. From these, a model of the heat output was developed that matches the heating rate inferred from the helium tank temperature during a cryogen mode recycle. As a result, the total heat output of the ADR can be used as a mass gauge tool during the mission. In addition, the analysis confirmed that the ADR's time average heat load to the liquid helium is $0.081-0.115 \mathrm{~mW}$, depending on the recycling interval. The lowest heat load corresponds to recycling at the end of a hold time, and the higher value corresponds to recycling every 24 hours.

In cryogen-free mode, two of the ADR's stages are used to continuously cool the helium tank to $\sim 1.5 \mathrm{~K}$. Their cooling power must exceed the parasitic heat load on the tank plus dissipation within the ADR so that the stages build cooling capacity that is used to recycle the $50 \mathrm{mK}$ stage. The theoretical heat lift capability in the $1.5-1.6 \mathrm{~K}$ range is $3.3-3.8 \mathrm{~mW}$. The tank's parasitic load averages $0.65 \mathrm{~mW}$. Internal dissipation from hysteresis in the magnets, power to the heat switch, and heat transfer losses adds 0.79 $\mathrm{mW}$. The stages generate an additional $0.45-0.59 \mathrm{~mW}$ of cooling that gets stored in Stage 2. Combined, the ADR absorbs 1.8-2.05 $\mathrm{mW}$ at $1.5-1.6 \mathrm{~K}$, and rejects an average of $8.7 \mathrm{~mW}$ to $4.5 \mathrm{~K}$ (the peak transfer rate is required to be less than $30 \mathrm{~mW}$ ). Subtracting out the internal losses gives a "useful" cooling power of 1.14-1.30 mW, and an efficiency (not counting electronics power dissipation) of 37-40\%.

\section{ACKNOWLEDGMENTS}

The work was supported by NASA's Office of Space Science.

\section{REFERENCES}

\footnotetext{
${ }^{1}$ Mitsuda, K, et al., 2010, “The high-resolution x-ray microcalorimeter spectrometer system for the SXS on ASTRO-H”, Proc. SPIE 7732, Space Telescopes and Instrumentation 2010: Ultraviolet to Gamma Ray, pp. 773211-773211-10.
} 
${ }^{2}$ Takahashi, T, et al., 2010, “The ASTRO-H Mission”, Proc. SPIE 7732, Space Telescopes and Instrumentation 2010: Ultraviolet to Gamma Ray, pp. 77320Z-77320Z-18.

${ }^{3}$ Peter J. Shirron, Mark O. Kimball, Bryan L. James, Donald C. Wegel, Raul M. Martinez, Richard L. Faulkner, Larry Neubauer, Marcelino Sansebastian, Design and predicted performance of the 3-stage ADR for the Soft-X-ray Spectrometer instrument on Astro-H, Cryogenics, Volume 52, Issues 4-6, April-June 2012, Pages 165-171

${ }^{4}$ Ryuichi Fujimoto, et al., Cooling system for the soft X-ray spectrometer onboard Astro-H, Cryogenics, Volume 50, Issue 9, September 2010, Pages 488-493

${ }^{5}$ Peter J. Shirron, Applications of the magnetocaloric effect in single-stage, multi-stage and continuous adiabatic demagnetization refrigerators, Cryogenics, Volume 62, July-August 2014, Pages 130-139

${ }^{6}$ Kelley RL, et al., The Suzaku High Resolution X-Ray Spectrometer, Publ. Astron. Soc. Japan 59, S77-S112, 2007 January 25

${ }^{7}$ Peter Shirron, Mark Kimball, Bryan James, Theo Muench, Michael DiPirro, Tom Bialas, Gary Sneiderman, Scott Porter, Richard Kelley, Operating Modes and Cooling Capabilities of the 3-Stage ADR Developed for the Soft-X-ray Spectrometer Instrument on Astro-H, Cryogenics, submitted for publication in this special issue

${ }^{8}$ Ventura G, Barucci M, Gottardi E, and Peroni I, Low temperature thermal conductivity of Kevlar, Cryogenics 40 (2000) 489-491.

${ }^{9}$ Bryan L. James, Raul M. Martinez, Peter Shirron, Jim Tuttle, John J. Francis, Marcelino San Sebastian, Donald C. Wegel, Nicholas M. Galassi, Daniel S. McGuinness, David Puckett, Yury Flom, Mechanical design of a 3-stage ADR for the Astro-H mission, Cryogenics, Volume 52, Issues 4-6, April-June 2012, Pages 172-177

${ }^{10}$ Wikus P, Hertel SA, Leman SW, McCarthy KA, Ojeda SM, and Figueroa-Feliciano E, The electrical resistance and thermal conductivity of $\mathrm{Ti} 15 \mathrm{~V}-3 \mathrm{Cr}-3 \mathrm{Sn}-3 \mathrm{Al}$ at cryogenic temperatures, Cryogenics 51 (2011) 41-44

${ }^{11}$ Kimball M, Shirron P. Heat Switches Providing Low-Activation Power and Quick-Switching Time For Use In Cryogenic Multi-Stage Refrigerators. In: Advances In Cryogenic Engineering, Vols 57A AND 57B. Joint Conference on Transactions of the Cryogenic Engineering Conference (CEC)/International Cryogenic Materials Conference (ICMC); JUN 13-17, 2011; Spokane, WA. 2012. p. 853-858

${ }^{12}$ Hagmann C, Richards PL. Specific heat of stainless steel below T =1K, Cryogenics 1995 Volume 35 p. 345

${ }^{13}$ Peter J. Shirron, Dan McCammon, Salt pill design and fabrication for adiabatic demagnetization refrigerators, Cryogenics, Volume 62, July-August 2014, Pages 163-171

${ }^{14}$ Kimball MO and Shirron PJ, Fast-response, active gas-gap heat switches for low temperature applications, submitted for publication in the proceedings of the 2015 Cryogenics Engineering Conference, Tucson AZ (2015).

${ }^{15}$ Source of supply: Supercon, Inc., 830 Boston Turnpike, Shrewsbury, MA 01545

${ }^{16}$ M.J. DiPirro, P.J. Shirron and J. G. Tuttle, "Mass Gauging and Thermometry on the Superfluid Helium On-Orbit Transfer Flight Demonstration", Adv. Cryo. Eng., 39, 129 (1994).

${ }^{17}$ Yoshida S, et al., Flight model performance test results of a helium dewar for the soft X-ray spectrometer onboard ASTRO-H, Cryogenics, submitted for publication in this issue 